\title{
Gram negatives get the cleaners in
}

Julio Ramos Aires and Hiroshi Nikaido have obtained biochemical evidence that a multidrug efflux pump in Gram-negative bacteria functions as a 'periplasmic vacuum cleaner, according to results published in the Journal of Bacteriology.

Bacteria encode various multidrug resistance (MDR) efflux pumps that actively extrude molecules, including antibiotics, out of cells. Of the five main families of MDR pumps, members of the resistance-nodulation-division (RND) family are among the most studied. In Gram-negative bacteria, MDR pumps are tripartite protein complexes that comprise inner membrane, periplasmic and outer membrane components, ensuring that substrates can be transported from the cytoplasm direct to the external milieu. Early intact-cell studies with two prototypical RND pumps - AcrAB-TolC and MexAB-OprM - combined with structural information indicated that substrates can also diffuse laterally from the outer leaflet of cytoplasmic membrane or periplasm. However, until now, biochemical evidence for this hypothesis has been elusive.

Aires and Nikaido used reconstituted proteoliposomes (sealed, phospholipid-bound vesicles) containing AcrD, an AcrB homologue. AcrD was used instead of AcrB, as the aminoglycoside substrates for AcrD, such as gentamicin, are highly charged and therefore unable to cross the vesicle membrane spontaneously, unlike the lipophilic substrates of AcrB. The addition of the natural ionophore valinomycin creates an artificial proton gradient, so that the $\mathrm{pH}$ inside the vesicles is more acidic than the extravesicular $\mathrm{pH}$, therefore mimicking the periplasm and cytoplasm, respectively.

When substrate is captured by AcrD and pumped into the vesicles (extruded from the cytoplasm to the periplasm), the corresponding counter-movement of protons should ensure that the intravesicular $\mathrm{pH}$ returns to alkaline faster than it would by spontaneous proton leakage. However, in the initial assays no enhanced efflux of protons was observed. Further assays revealed that the activity of AcrD was dependent on the presence of the periplasmic membrane fusion protein (MFP) AcrA. Additionally, the presence of gentamicin within the vesicles (in the periplasm) enhanced proton move-

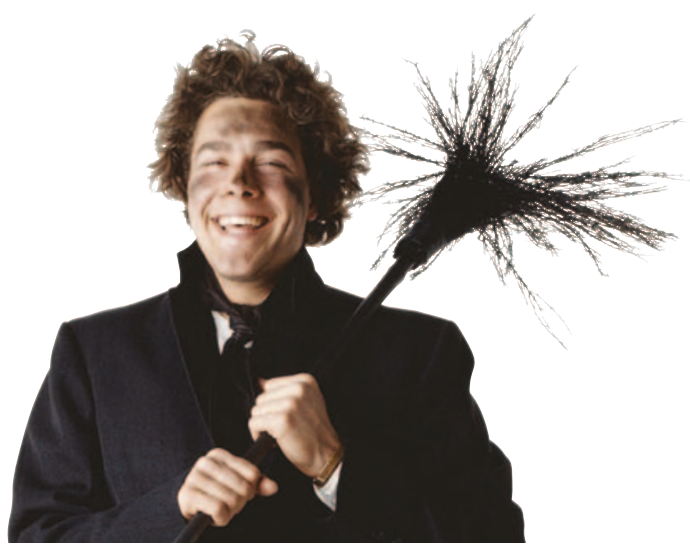

ment by AcrD in the presence of AcrA, indicating that substrate can be captured from the periplasm.

Previously, MFPs have generally been ascribed passive roles in substrate transport by MDR pumps. In this work, Aires and Nikaido have not only provided biochemical evidence for the long-held hypothesis of periplasmic substrate capture, they have also uncovered an active role for an MFP. They propose that AcrA activates AcrD, and that the AcrAAcrD complex then acts as antiporter, coupling the efflux of protons to the influx of aminoglycosides.

Sheilagh Molloy

(2) References and links ORIGINAL RESEARCH PAPER Aires, J. R. \& Nikaido, H. Aminoglycosides are captured from both periplasm and cytoplasm by the AcrD multidrug efflux transporter of Escherichia coli. J. Bacteriol. 187, 1923-1929 (2005)

FURTHER READING Lomovskaya, O. \& Totrov, M. Vacuuming the periplasm. J. Bacteriol. 187, 1879-1883 (2005) 